\title{
Observations of weak boson scattering with the ATLAS detector
}

\section{Takuya Nobe ${ }^{a, *}$ on behalf of the ATLAS Collaboration}

a International Center for Elementary Particle Physics, the University of Tokyo, 7-3-1 Hongo, Bunkyo-ku, Tokyo, Japan

E-mail: Takuya.Nobe@cern.ch

Vector-boson scattering (VBS) is one of the most important physics processes at the Large Hadron Collider for studying the mechanism of electroweak symmetry breaking. This article presents the latest measurements of the electroweak diboson with two jets productions, including VBS processes, by the ATLAS experiment using $p p$ collisions data at $\sqrt{s}=13 \mathrm{TeV}$ corresponding to integrated luminosities of $36-139 \mathrm{fb}^{-1}$. The same-sign $W^{ \pm} W^{ \pm} j j$ and $Z Z j j$ productions are observed with greater than $5 \sigma$ accuracies. The $Z \gamma j j$ production and the inclusive electroweak $V V j j$ production in semi-leptonic final states are measured with $4.2 \sigma$ and $2.7 \sigma$ standard deviations from the background-only hypothesis, respectively. The results are compared with several theoretical predictions and good agreements with the Standard Model are reported.

40th International Conference on High Energy physics - ICHEP2020

July 28 - August 6, 2020

Prague, Czech Republic (virtual meeting)

\footnotetext{
${ }^{*}$ Speaker
} 


\section{Introduction}

Vector-boson scattering (VBS) is one of the most important physics processes at the Large Hadron Collider for studying the mechanism of electroweak symmetry breaking. In addition, some new physics models beyond the Standard Model (SM), that alter the quartic gauge couplings or include unknown high-mass diboson resonances, predict to enhance VBS cross sections at the high invariant mass of the diboson system. Hence it is important to measure the VBS cross sections in a wide mass range, using all possible processes model-independently, such as $W W, W Z$ and $Z Z$ scattering, collectively referred to as $V V$ scattering $(V=W$ or $Z$ ), as well as $V \gamma$ and $\gamma \gamma$ scattering. As shown in Figure 1 (a), the experimental feature of the VBS processes is the existence of high transverse momentum $\left(p_{\mathrm{T}}\right)$ quark-induced hadron jets in the forward-backward directions and two $W, Z$ or $\gamma$ bosons in the central rapidity. In most analysis channels, the main source of background is the QCD-induced diboson production in association with two jets as shown in Figure 1 (b).

This article presents the recent ATLAS measurements of the electroweak diboson productions, including VBS processes, using the Run-2 $p p$-collisions datasets at $\sqrt{s}=13 \mathrm{TeV}$, corresponding to $36-139 \mathrm{fb}^{-1}$ of integrated luminosities. Details of the ATLAS detector are found elsewhere [1].

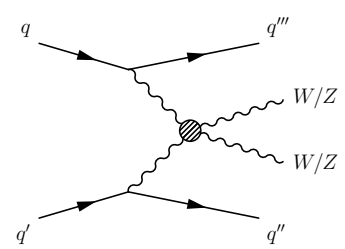

(a)

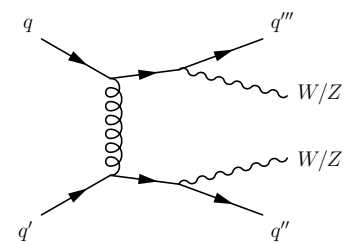

(b)

Figure 1: (a) VBS and (b) QCD-induced diagrams as representatives for diboson with two jets productions.

\section{Observation of the electroweak $W^{ \pm} W^{ \pm} \rightarrow \ell^{ \pm} v \ell^{ \pm \prime} v$ production}

The same-sign $W$-boson pair with two jets production $\left(W^{ \pm} W^{ \pm} j j\right)$ has the largest ratio of electroweak to QCD-induced production cross sections compared with other VBS processes. The analysis using the $36 \mathrm{fb}^{-1}$ dataset collected in years 2015-16 is found in Ref. [2]. Events are required to have exactly two same-sign isolated leptons (electron or muon) and a large missing transverse momentum $\left(E_{\mathrm{T}}^{\mathrm{miss}}\right)$. They should contain at least two high- $p_{\mathrm{T}}$ hadron jets in $|\eta|<4.5$. To enhance the VBS signal, a signal region (SR) is defined by $m_{j j}>500 \mathrm{GeV}$ and $\left|\Delta y_{j j}\right|>2.0$, where $m_{j j}$ and $\Delta y_{j j}$ are the invariant mass and the rapidity difference of the two highest- $p_{\mathrm{T}}$ jets. The signal yield is estimated by the fit to the $m_{j j}$ distribution as shown in Figure 2. The normalizations of $W Z$ and $V \gamma$ backgrounds are determined in the dedicated control regions (CRs) requiring three leptons and two opposite-sign muons plus high- $p_{\mathrm{T}}$ isolated photon, respectively. Background events containing non-prompt leptons or electric charge mis-identified electrons are estimated by data-driven methods. The normalization of QCD-induced $W^{ \pm} W^{ \pm} j j$ background is constrained by the data in the low- $m_{j j} \mathrm{CR}$ requiring $200<m_{j j}<500 \mathrm{GeV}$. A total of 122 candidate events are observed in the SR for a background expectation of $69 \pm 7$, corresponding to an observed signal significance of 6.5 standard deviations. The result is compared with the theoretical calculations by 


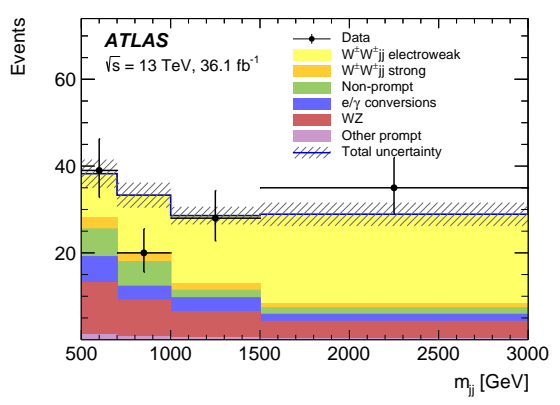

Figure 2: The number of data events, and signal and background events as predicted after the fit in bins of $m_{j j}$ for events passing all selection criteria of the signal region [2]. The hatched band represents the statistical and systematic uncertainties of the background prediction added in quadrature. The last bin of the distribution includes the overflow.

Sherpa 2.2.2 [3] (LO in QCD) and Powheg+Pythia8 [4, 5] (NLO) generators. Data agree very well with the Powheg +Pythia8 prediction and the ratio is $0.94_{-0.16}^{+0.17}$ (stat.) \pm 0.17 (syst.), while the ratio to the SHERPA prediction is $1.44_{-0.24}^{+0.26}$ (stat.) ${ }_{-0.22}^{+0.28}$ (syst.), where uncertainties correspond to statistical and systematic components. The data are consistent with both generators within about $1 \sigma$, but they predict different fiducial cross sections. The dominant source of the uncertainty on the fiducial cross section prediction for the SHERPA sample is the choice of the QCD scales, while PDF and parton shower modeling uncertainties are important for PowHEG+PyTHIA8.

\section{Observation of the electroweak $Z Z$ with two jets in the $4 \ell$ and $\ell \ell v v$ final states}

The electroweak $Z Z j j$ production has the smallest cross section among the $V V j j$ processes in the SM. Using the full Run-2 dataset corresponding to $139 \mathrm{fb}^{-1}$, the first observation of this process has been achieved [6]. Two different final states, $Z Z \rightarrow 4 \ell$ and $Z Z \rightarrow \ell \ell v v$, are employed. In the $4 \ell$ channel, SR is defined by the existence of lepton quadruplets formed by two opposite-sign and same-flavor (OSSF) lepton pairs, with the masses consistent with the Z-boson mass. In the $\ell \ell v v$ channel, events in SR are required to have one OSSF lepton pair and large $E_{\mathrm{T}}^{\text {miss }}$-significance calculated using resolution information of physics objects used in the $E_{\mathrm{T}}^{\text {miss }}$ reconstruction. In both channels, two jets in $|\eta|<4.5$ with the highest $p_{\mathrm{T}}$ and satisfying a negative product of rapidities are selected. The $m_{j j}$ is required to be greater than $300(400) \mathrm{GeV}$ in the $4 \ell(\ell \ell v v)$ channel, and $\left|\Delta y_{j j}\right|$ to be greater than 2.0. After the event selections, the main source of the background in the $4 \ell$ channel is QCD-induced $Z Z j j$ production (about 70\%). In the $\ell \ell v v$ channel, contributions from QCD-induced $Z Z j j$, non-resonant dilepton $(t \bar{t}, W W j j$ etc.) and $W Z$ processes are $20-25 \%$ each. The normalization of QCD-induced $Z Z j j$ in the $4 \ell$ channel is constrained by the data in the CR reversing either the $m_{j j}$ or $\Delta y_{j j}$ cuts. The impact on the signal extraction of a potential mismodelling of the $m_{j j}$ distribution in the QCD-induced $Z Z j j$ simulation is studied and found to be smaller than the statistical uncertainty. The normalization of non-resonant dilepton background is estimated in the CR requiring different flavor lepton pair $(e \mu)$. The normalization of $W Z$ background is estimated in the three-lepton CR.

To enhance the electroweak signal more, a multi-variate analysis based on Boosted Decision Trees (BDT) is introduced. In each channel, a BDT is trained in SR, which uses event kinematic information sensitive to the characteristics of the signal, to separate the electroweak $Z Z j j$ from the background. Twelve (thirteen) input variables are used in the $4 \ell(\ell \ell v v)$ channel. As shown in Figure 3, the signal yields are estimated by the fit to the BDT score distributions in both channels simultaneously. The hypothesis of no electroweak production is rejected with a statistical 


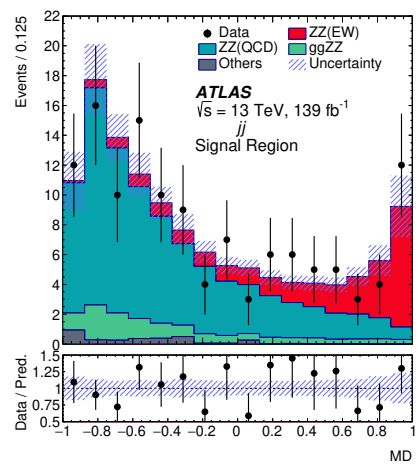

(a)

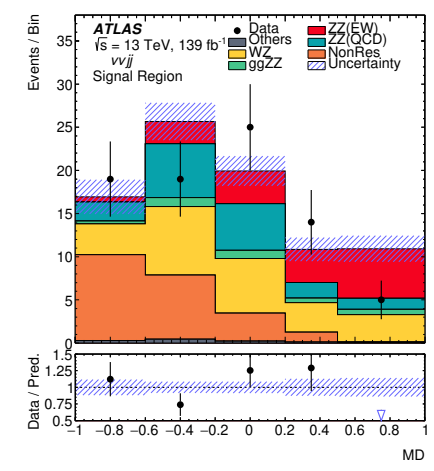

(b)

Figure 3: Observed and expected multivariate discriminant (MD) distributions after the statistical fit in the (a) $4 \ell$ and (b) $\ell \ell v v$ SRs [6]. The error bands include the experimental and theoretical uncertainties. All the minor backgrounds are summed together as 'Others. The statistical uncertainties of the data are shown as error bars. The open arrows represent the out-of-range markers.

significance of $5.5 \sigma$. The ratio of the observed signal yield in the fiducial phase space to the theoretical prediction by the MADGRAPH 2.6.1 [7] event generator (LO) is $1.35 \pm 0.34$ (stat.+syst.).

\section{Evidence of $Z \gamma$ scattering in dilepton final state mode}

The $Z \gamma$ scattering is interesting because it allows the measurement of the neutral quartic gauge couplings, as for the $Z Z$ final state but with a larger expected cross section. The analysis using the $36 \mathrm{fb}^{-1}$ dataset collected in 2015-16 is reported in Ref. [8]. Events are required to have exactly one OSSF isolated lepton pair and at least one high- $p_{\mathrm{T}}$ isolated photon. The invariant mass of the lepton pair is required to be comparable with the $Z$-boson mass. Two jets in $|\eta|<4.5$ with the highest $p_{\mathrm{T}}$ and satisfying a negative product of rapidities are selected. The $m_{j j}$ is required to be greater than $150 \mathrm{GeV}$ and $\left|\Delta \eta_{j j}\right|$ to be greater than 1.0 , where $\Delta \eta_{j j}$ is the difference of pseudo-rapidities of the two jets. Finally, SR is defined by requiring the centrality of the $\ell \ell \gamma$ system relative to the tagging jets: $\zeta(\ell \ell \gamma)=\left|\frac{y_{\ell \ell}-\left(y_{j 1}+y_{j 2}\right) / 2}{y_{j 1}-y_{j 2}}\right|$ to be less than 5.0, where $y$ corresponds to the rapidity and $j 1$ and $j 2$ label the selected leading and subleading jets.

A BDT is trained to enhance the electroweak signal more, using thirteen kinematic variables. The signal yield is estimated by the fit to the BDT score distribution. The normalization of QCD-induced $Z \gamma$ production is estimated by the fit at the same time in lower BDT bins. The $\mathrm{CR}$ requiring additional $b$-tagged jets is added to the fit to give a constraint on the normalization of the $t \bar{t}+\gamma$ background. The background with one hadron jet misidentified as a photon is estimated by a dedicated data-driven method. Figure 4 shows the BDT score distribution in SR after the fit. The observed signal yield corresponds to a statistical significance of 4.1 standard deviations. The ratio to the theoretical prediction by the MADGRAPH 2.3.3 (LO) event generator is $1.00 \pm 0.19$ (stat.) \pm 0.13 (exp.) ${ }_{-0.10}^{+0.13}$ (mod.), where "exp." and "mod." denote the experimental (e.g. jet energy scale) and the theoretical sources of the systematic uncertainty. The observed ratio to SHERPA 2.4.4 (LO) prediction is $0.87 \pm 0.17$ (stat.) \pm 0.11 (exp.) \pm 0.12 (mod.). 


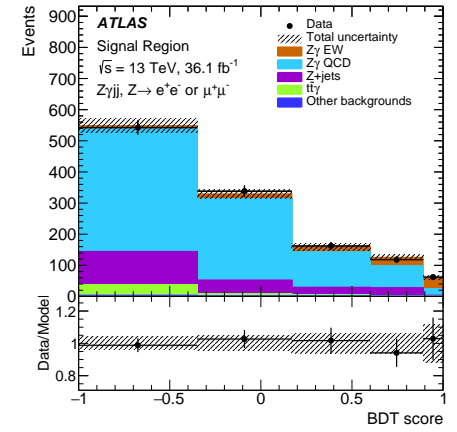

Figure 4: Post-fit distributions of the BDT score in SR [8]. The uncertainty band around the expectation includes all systematic uncertainties.

\section{Search for $W W / W Z / Z Z$ scattering in the semi-leptonic final states}

The hadronic decay of $W / Z$ boson has the highest branching fraction and it is useful for the measurement of VBS processes at the TeV energy scale. The electroweak $V V j j$ production in the "semi-leptonic" final state modes, where one of the bosons decays leptonically and the other hadronically ( $W V \rightarrow \ell v q q$ and $Z V \rightarrow \ell \ell q q / v v q q$ ), have been measured using $36 \mathrm{fb}^{-1} p p$ collisions data collected in 2015-16 [9]. Since the new physics contribution is expected to be larger in the high-mass region, this channel can give the strongest limit on some models.

Depending on the target mass range, two analysis categories are defined. In the lower-mass region, two quarks from the boson decay can be resolved by the standard jet algorithm in ATLAS (anti- $k_{t}$ with the radius parameter $R=0.4$ ). In the higher-mass region, they are merged together into a single large-radius jet $(R=1.0)$ and the "boosted boson tagging" technique using jet substructure is employed to enhance the signals. In both categories, a window cut on the reconstructed hadronicallydecaying boson candidate mass is applied to be compatible with $W / Z$ mass. An additional pair of jets is selected in $|\eta|<4.5$ with the highest- $m_{j j}$ and negative product of pseudo-rapidities, excluding the jets used in $W / Z \rightarrow q q$ reconstruction. The $m_{j j}$ is required to be greater than $400 \mathrm{GeV}$. Nine SRs are involved in the fit, defined by the number of leptons and the categories of hadronicallydecaying $W / Z$ reconstruction. The main source of the background is single $W / Z$ boson production in association with hadron jets $(V+$ jets). The contribution from $t \bar{t}$ is also significant in $\ell v q q$ and $v v q q$ final states. The normalization of $V+$ jets background is constrained by the CRs reversing the $W / Z \rightarrow q q$ mass window cut. The normalization of the $t \bar{t}$ background is constrained by the CRs requiring additional $b$-tagged jets in the $\ell v q q$ final state. The modeling of the $m_{j j}$ distribution in the $V+$ jets simulated events is corrected by data in the control sample and a conservative uncertainty is assigned in the correction factors.

In each SR, a BDT is trained to enhance the electroweak signal against the background. Four to sixteen kinematic variables are used as inputs. The signal yields are estimated by the fit to the BDT score distributions in all SRs simultaneously. Figure 5 shows examples of the post-fit BDT score distributions. The ratio of the observed fiducial cross section to the MADGRAPH 2.4.3 prediction (LO) is $1.05 \pm 0.20$ (stat.) ${ }_{-0.34}^{+0.37}$ (syst.). The background only hypothesis is excluded with a statistical significance of $2.7 \sigma$. The main source of systematic uncertainty comes from the correction factor of the $m_{j j}$ distribution in the $V+$ jets simulated events. 


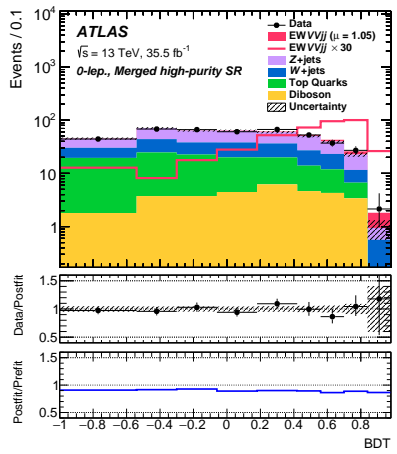

(a)

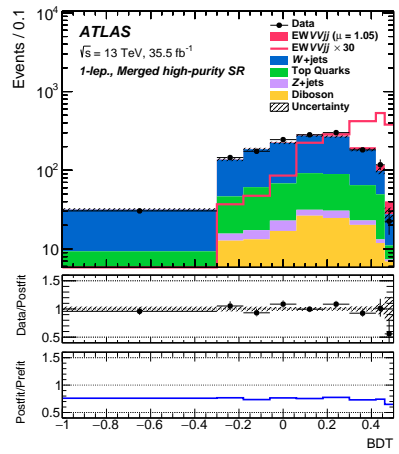

(b)

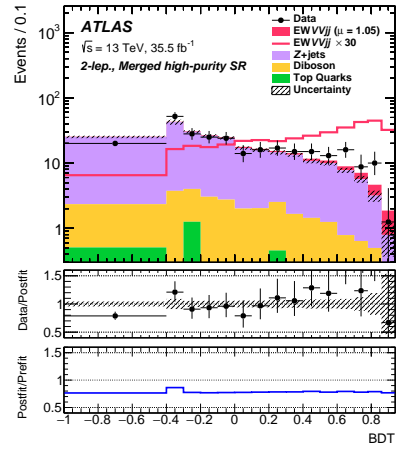

(c)

Figure 5: Examples of the post-fit BDT score distributions in (a) $v v q q$, (b) $\ell v q q$ and (c) $\ell \ell q q$ SRs [9]. The middle panel shows the ratios of the observed data to the signal plus background predictions. The uncertainty shown by the band includes both statistical and systematic contributions. The bottom panel shows the ratios of the post-fit and pre-fit background predictions.

\section{Conclusions}

The recent ATLAS measurements of the electroweak $V V j j$ productions have been reported, using $13 \mathrm{TeV} p p$ collisions data at the Large Hadron Collider corresponding to integrated luminosities of 36-139 $\mathrm{fb}^{-1}$. The same-sign $W^{ \pm} W^{ \pm} j j$ and $Z Z j j$ processes are observed with greater than $5 \sigma$ accuracies. The $Z \gamma$ process is measured with $4.2 \sigma$. The inclusive electroweak $V V j j$ cross section is measured in semi-leptonic final state modes. The observed statistical significance is found to be $2.7 \sigma$. The results are compared with several theoretical predictions and good agreements with the Standard Model are reported.

\section{References}

[1] ATLAS Collaboration, JINST 3 (2008) S08003.

[2] ATLAS Collaboration, Phys. Rev. Lett. 123 (2019) 161801.

[3] T. Gleisberg et al., JHEP 02 (2009) 007.

[4] P. Nason, JHEP 11 (2004) 040.

[5] T. Sjöstrand et al., Comput. Phys. Commun. 191 (2015) 159.

[6] ATLAS Collaboration, submitted to Nature Physics, arXiv: 2004.10612[hep-ex].

[7] J. Alwall et al., JHEP 07 (2014) 079.

[8] ATLAS Collaboration, Phys. Lett. B 803 (2020) 135341.

[9] ATLAS Collaboration, Phys. Rev. D 100 (2019) 032007. 